Draft VERSion September 4, 2020

Typeset using IATEX RNAAS style in AASTeX63

\title{
Rotation Optimized Filter for Longevity (ROFL): Increasing the lifetime of Swift/UVOT simply
}

\author{
Aaron Tohuvavohu (D) ${ }^{1}$ \\ ${ }^{1}$ Department of Astronomy \& Astrophysics \\ University of Toronto \\ Toronto, ON, Canada
}

Submitted to RNAAS

\begin{abstract}
I demonstrate that a very simple and safe change to the planning software filter assignment algorithm for the Ultraviolet/Optical Telescope (UVOT) onboard the Neil Gehrels Swift Observatory can reduce the number of filter wheel rotations by $>10 \%$, and its adoption is thus likely to significantly extend the usable lifetime of the UVOT instrument.
\end{abstract}

\section{INTRODUCTION}

The Ultra-Violet Optical Telescope (UVOT; Roming et al. (2005)) onboard the Neil Gehrels Swift Obsevatory (Gehrels et al. 2004) is a workhorse instrument of the time-domain community, and the only instrument regularly performing transient science and fast follow-up in the UV. The UVOT has a photon counting detector with a bandpass from 170-700 nm, covering a 17'x17' field-of-view. In the optical path is an 11-position filter wheel, carrying 7 filters, 2 grisms, a focal expander (magnifier), and a blocking plate. The filter wheel rotates on a stub axle, and is driven by a uni-directional stepper motor. The filter wheel was rated for 50,000 revolutions in its design, and has substantially surpassed this number to date. The UVOT filter wheel is the only regularly moving mechanism in any of the Swift instruments, and is likely to be the main lifetime limiting component for UVOT.

\section{FILTER-OF-THE-DAY}

For this reason, limiting and optimizing the number of rotations made by the filter wheel is paramount. The method adopted at the beginning of the Swift mission, and used still today, is the 'Filter of the Day' (FOTD) ${ }^{1}$ scheme: whereby each day is assigned one of the four near-UV filters, in a repeating cycle, and all observations not requiring a particular mode or filter set from UVOT (ie observations for which the main science is being performed with the XRT) are set in this filter. The near-UV (and not optical) filters are chosen so as to ensure there is still a unique UV contribution to the observation. Such an arrangement is designed to have as many consecutive observations be scheduled in the same filter as possible to eliminate the need for movement of the filter wheel.

At the beginning of the Swift mission the vast majority of observations were XRT driven, and the science cases required no specific UVOT filters or modes, thus allowing for a substantial number of consecutive observations with UVOT set in the FOTD, and proving the efficacy of this approach to reducing the filter wheel rotations. However, over the course of the Swift mission the UVOT has become a high-demand instrument for the community, for science ranging from comet observations in the inner solar system (Bodewits et al. 2011), near-UV exoplanet transits (Salz et al. 2019), the mapping of star formation histories of nearby galaxies (Hagen et al. 2017), and most predominantly, providing early-time UV light curves for $\sim 1000$ supernovae to date (Brown et al. 2014), along with myriad other transients (eg the first UV detection of a kilonova Evans et al. (2017)). The success of the community in fully utilizing UVOT, as well as increased demand on Swift overall, has changed the typical Swift schedule. While the FOTD technique is still used when appropriate, the majority of science programs require specific UVOT filters and modes,

Corresponding author: Aaron Tohuvavohu

tohuvavohu@astro.utoronto.ca

${ }^{1}$ https://www.swift.psu.edu/secure/toop/uvot_filter_rotation_info.htm 
and thus the number of consecutive observations in FOTD are significantly reduced. In addition the Swift schedule has grown denser, with more individual observations performed on average per day each year (> 100 per day in 2019).

\section{AN ALTERNATIVE}

Under these substantially different scheduling conditions for UVOT, and in the context of larger advancements in Swift scheduling (Tohuvavohu 2018; Deich et al. 2018), I examined the efficacy of the FOTD scheme for reducing filter wheel rotations and found that it substantially under-performed with respect to a simple alternative:

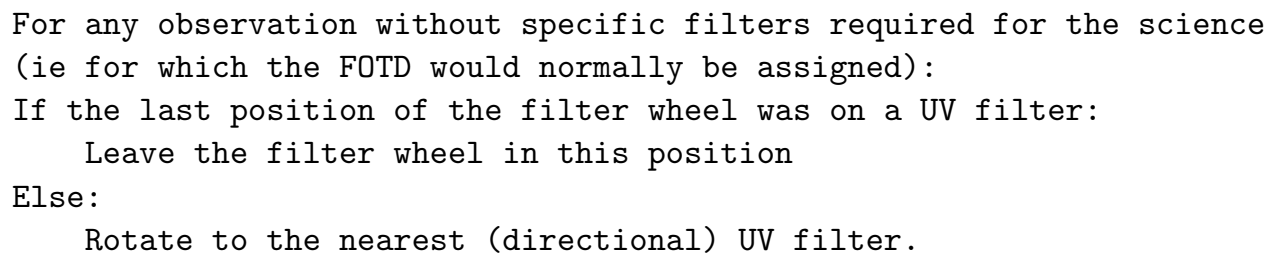

I rebuilt the Swift observing schedules for all of 2018 and 2019 using this schema, and find a reduction of $>18,000$ filter wheel movements per year as compared to the FOTD scheme. This corresponds to a $>10 \%$ reduction in the number of rotations performed, and thus likely a similar extension to the lifetime of the filter wheel. This new scheme, which I call the Rotation Optimized Filter for Longevity (ROFL), naturally entails a different distribution in the use of the UV filters, which I show in Figure 1:

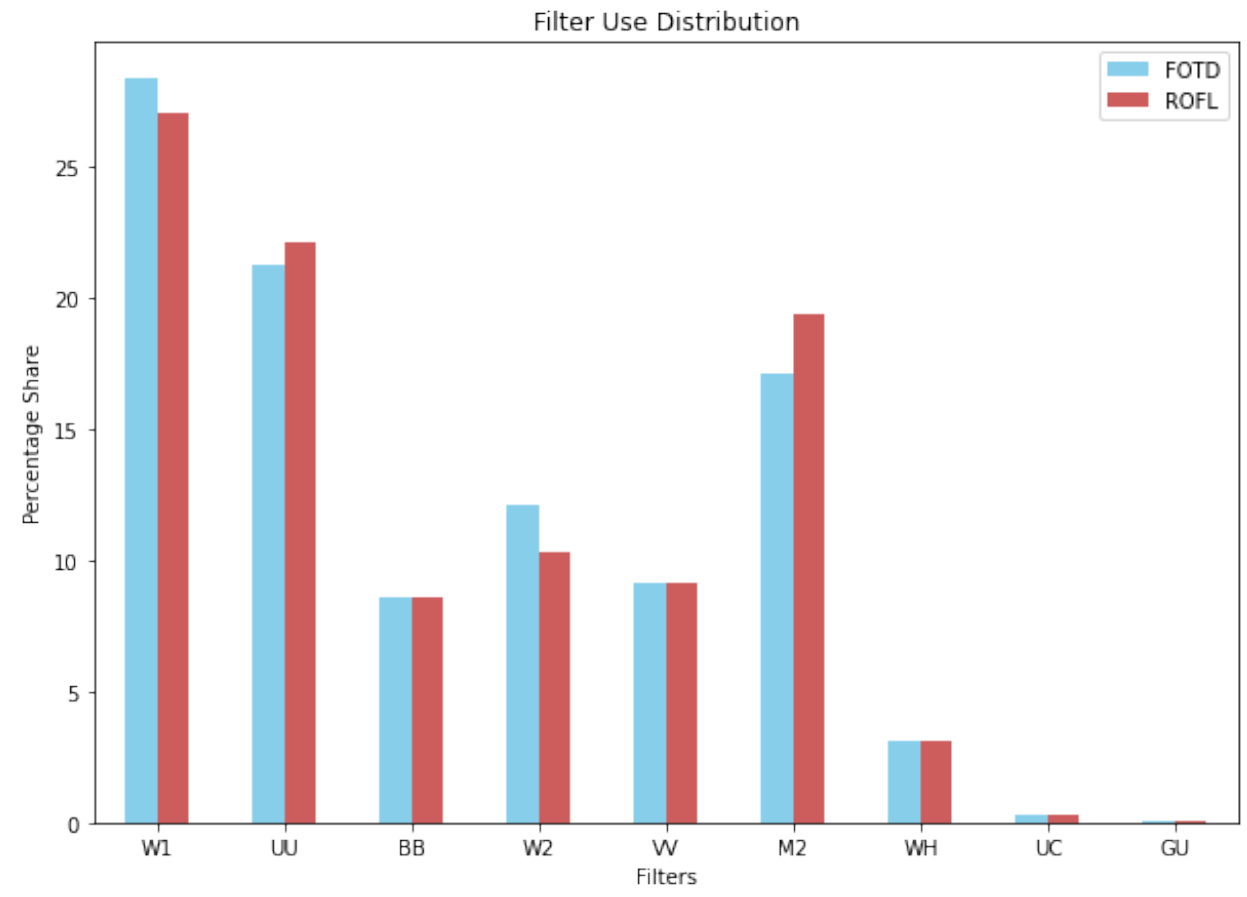

Figure 1. The filter use distribution of all Swift/UVOT observations scheduled in 2019. Shown for both the FOTD scheme, and the proposed ROFL scheme. ROFL results in a slight increase in the number of observations taken with the $u$ and $u v m 2$ filters, and a corresponding decrease in the use of the $u v w 1$ and $u v w 2$ filters.

Given the simplicity of this scheme and the large impact it can have on reducing the wear on the UVOT filter wheel, and therefore likely increasing the functional lifetime of the instrument, I recommend that it be implemented within the Swift planning software.

\section{REFERENCES}

Bodewits, D., Villanueva, G. L., Mumma, M. J., et al. 2011, AJ, 141, 12, doi: 10.1088/0004-6256/141/1/12
Brown, P. J., Breeveld, A. A., Holland, S., Kuin, P., \& Pritchard, T. 2014, Ap\&SS, 354, 89, doi: 10.1007/s10509-014-2059-8 
Deich, A., Gropp, J., \& LaPorte, S. 2018, in Society of Photo-Optical Instrumentation Engineers (SPIE) Conference Series, Vol. 10699, Space Telescopes and Instrumentation 2018: Ultraviolet to Gamma Ray, 106996C, doi: 10.1117/12.2320320

Evans, P. A., Cenko, S. B., Kennea, J. A., et al. 2017, Science, 358, 1565, doi: 10.1126/science.aap9580

Gehrels, N., Chincarini, G., Giommi, P., et al. 2004, ApJ, 611, 1005, doi: 10.1086/422091

Hagen, L. M. Z., Siegel, M. H., Hoversten, E. A., et al. 2017, MNRAS, 466, 4540, doi: 10.1093/mnras/stw2954
Roming, P. W. A., Kennedy, T. E., Mason, K. O., et al. 2005, SSRv, 120, 95, doi: 10.1007/s11214-005-5095-4

Salz, M., Schneider, P. C., Fossati, L., et al. 2019, A\&A, 623, A57, doi: 10.1051/0004-6361/201732419

Tohuvavohu, A. 2018, in Journal of Physics Conference Series, Vol. 1085, Journal of Physics Conference Series, 032010, doi: 10.1088/1742-6596/1085/3/032010 